\title{
Aprendizagem baseada em projetos aplicada em uma disciplina de integração de Engenharias: desafios e benefícios
}

\author{
Ricardo Ajax Dias Kosloski, Cristiane Soares Ramos \\ Edna Dias Canedo, Helena Bretas Goulart
}

\author{
${ }^{1}$ Universidade de Brasília (UnB) - Brasília-DF, Brasil \\ rikosdf@gmail.com, cristianesramos@unb.br, ednacanedo@unb.br, \\ helenabretasg@gmail.com
}

\begin{abstract}
This paper presents an experience case report about the application of Project-Based-Learning (PBL) in an integrational engineering discipline, offered in an engineering faculty at University of Brasília $(U n B)$ during 3 consecutive semesters. Moreover, is presented a set of lessons learned, barriers and facilitators found in the implementation of $P B L$, in the perspective of teachers and students. Among the various results, it can be highlighted the difficulties of interaction between students of different engineering and the importance of the teacher's guidance in case of the occurrences of technical problems in the assembly of products sought by the projects. The use of PBL was considered appropriate to facilitate the aggregation of technical and human aspects, involving students and teachers among 5 distinct engineering courses, there are in the Faculty.
\end{abstract}

Resumo. Este artigo apresenta um relato de experiência da aplicação da Aprendizagem Baseada em Projetos (ABP) em uma disciplina integradora entre as Engenharias ofertadas na Faculdade FGA da Universidade de Brasília $(U n B)$ durante 03 semestres consecutivos. Além disso, é apresentado um conjunto de lições aprendidas, barreiras e facilitadores encontrados na aplicação da ABP sob a perspectiva dos professores e estudantes. Dentre os vários resultados, podem ser ressaltadas as dificuldades em termos de delimitação de escopo dos projetos envolvendo todas as engenharias da faculdade, dificuldades próprias da interação entre os estudantes de diferentes engenharias e a importância das orientações dos professores quando da ocorrência de problemas técnicos na montagem dos produtos almejados pelos projetos. O uso da ABP foi considerada oportuna por viabilizar a agregação dos aspectos técnicos e humanos nos projetos, envolvendo alunos e professores nos cursos de 05 engenharias distintas.

\section{Introdução}

A Aprendizagem Baseada em Projetos (ABP) é uma abordagem que usa a construção de projetos como ferramenta pedagógica [Thomas 2000], [Blumenfeld et al. 1991], [Helle et al. 2006]. O conceito de projetos baseados na ABP é definido por meio de três tipos, a saber: os exercícios de projetos, que são aqueles onde a centralidade do ensino está no professor, os componentes de projeto e as orientações ao projeto, que são aqueles onde uma maior autonomia é dada ao aluno [Morgan 1975]. O principal representante do 
VIII Congresso Brasileiro de Informática na Educação (CBIE 2019)

Anais do XXX Simpósio Brasileiro de Informática na Educação (SBIE 2019)

primeiro tipo (exercícios de projetos), são os projetos que normalmente são chamados de Trabalho de Conclusão de Curso (TCC) ou Trabalho de Graduação (TG).

A definição dos exercícios de projetos é mais consolidada na literatura. No entanto, os outros dois tipos (componentes de projeto e orientações ao projeto) são tratados como pertencentes ao movimento progressista de educação fundamentado nos princípios construtivistas, que incentivam as abordagens que sejam orientadas ao aluno e produzem as habilidades para o século 21 [Bender 2012, Condliffe et al. 2017]. A diferença entre esses dois tipos é que os componentes de projeto são um tipo interdisciplinar de projeto e resolução de problemas que acontece em paralelo com outras disciplinas regulares e as orientações ao projeto tratam das abordagens que envolvem toda a comunidade escolar/acadêmica [Morgan 1975].

Este artigo apresenta um relato de experiência dos trabalhos realizados em uma disciplina integradora entre as Engenharias da Faculdade UnB Gama da Universidade de Brasília (UnB), os quais podem ser classificados como do tipo componentes de projeto e orientações ao projeto e possuem como objetivo principal a integração entre essas Engenharias, permitindo o compartilhando de conhecimento entre docentes e alunos e a busca por soluções que podem ser utilizadas pela comunidade em geral. Além disso, favorece a autonomia dos estudantes em propor soluções e serem conduzidos por profissionais experientes, apresentando para a comunidade em geral (alunos, docentes e colegas de turmas) suas ideias e soluções propostas.

A principal contribuição deste trabalho está no relato de como foi usada a ABP em uma disciplina visando a integração dos estudantes das Engenharias (Aeroespacial, Automotiva, Eletrônica, Energia e Software), a fim de estabelecer um projeto integrando esses estudantes para resolver um problema da vida real. O relato, além de identificar as fases e atividades do andamento da disciplina, também faz uma análise sobre os resultados obtidos sob o ponto de vista dos seus professores, relatando barreiras e facilitadores. Além disso foi incluída também uma perspectiva inicial da opinião dos estudantes sobre a disciplina. Os relatos são animadores e a aplicação da ABP forneceu motivação aos estudantes e professores, corroborando com as pesquisas que afirmam a sua vantagem em ambientes educacionais.

\section{Aprendizagem Baseada em Projetos e Trabalhos Correlatos}

Existem trabalhos na literatura que estabelecem limites para a prática da Aprendizagem Baseada em Projetos (ABP), estabelecendo características necessárias para que uma unidade de ensino se encaixe na definição de ABP. Assim sendo, existe uma série de características que delimitam um escopo do que poderia ser considerado projeto. Uma abordagem para ser considerada como ABP deve seguir os seguintes critérios [Thomas 2000, Condliffe et al. 2017]: 1) Ter projetos que são aderentes ao plano de ensino da disciplina; 2) Ser guiado por questões motrizes que podem motivar os alunos enquanto eles se envolvem na descoberta dos conceitos e princípios de uma determinada disciplina; 3) Permitir que os alunos trabalhem em uma investigação construtivista para transformação e construção do conhecimento; 4) Não se basear em projetos que são "empacotados" pelos professores, de forma que mais poder de escolha seja dado ao aluno; 5) Trabalhar com projetos ligados às questões do mundo real; 6) Prover um ambiente propício à colaboração entre os estudantes e professores; 7) Ser feita com base em uma 
VIII Congresso Brasileiro de Informática na Educação (CBIE 2019)

Anais do XXX Simpósio Brasileiro de Informática na Educação (SBIE 2019)

estratégia de andaimes (scaffolding), partindo de mais apoio a menos apoio aos alunos ao longo do tempo; 8) Ter como alvo objetivos de aprendizagem significantes, que ensinem os alunos habilidades úteis para sua vida profissional, mas não percam de vista os conteúdos obrigatórios das ementas.

A centralidade do projeto é um dos principais limites que determinam se uma abordagem pedagógica centrada no aluno poderá ser chamada de $\mathrm{ABP}$ ou não [Morgan 1975]. Além dessas características que um projeto deve ter para que a unidade letiva no qual ele está inserido possa ser chamada de ABP, o papel do professor também é colocado como um aspecto importante nessa caracterização. O poder de escolha e a motivação do aluno passaram a serem vistos como fatores propulsores para a aprendizagem por meio do "fazer" porque são eles que ativam a motivação dos alunos para o aprendizado [Knoll 1997]. Por causa disso, o papel de um professor dentro de uma unidade de $\mathrm{ABP}$ não deve ser o de um expositor de conteúdos, mas o de um facilitador, tutor ou coach que irá guiar o aluno durante a unidade de ABP [Moursund 1999], [Morgan 1983]. Sem essas características, a execução do "aprender fazendo" dentro de uma sala de aula pode ser chamado apenas de execução de tarefas e não de projetos educacionais [Knoll 1997, Bender 2012], [Jones et al. 1997], [Thomas et al. 1999], [Adderley et al. 1975], [Blumenfeld et al. 1991], [Helle et al. 2006], [Diehl et al. 1999], [Krajcik et al. 1994].

A aprendizagem baseada em projetos tem sido adotada em diversos contextos educacionais, incluindo no currículo de engenharias, quer para aumentar a motivação, quer para permitir que os estudantes apliquem na prática o que aprenderam [Martins et al. 2017]. A aprendizagem baseada em projetos permite aos estudantes investigar questões, propor hipóteses e explicações, discutir as suas ideias, estimular o aparecimento de novas ideias e experimentar essas ideias. Exige que os estudantes projetem e concluam projetos, em contextos que são confrontados com diversos problemas abrangentes para os quais precisam de encontrar soluções. A resolução de problemas é um processo demorado e, quando necessário, para completar uma tarefa em aprendizagem baseada em projetos, exige mais tempo do estudante do que métodos passivos de aprendizagem, porque os estudantes, geralmente, precisam de fazer várias tentativas antes de atingirem uma conclusão satisfatória [Mahasneh and Alwan 2018]. Os estudantes devem ser preparados para propor, investigar e desenvolver soluções para os problemas que possam surgir na sua vida profissional. Devem ser estimulados durante o seu curso a trabalhar com problemas com níveis crescentes de complexidade, o que exige dos professores uma mudança nas metodologias utilizadas. A metodologia de aprendizagem baseada em projetos permite desenvolver competências dos estudantes em diversos contextos [Mahasneh and Alwan 2018]. Os pilares chaves de unidades de ABP são [Condliffe et al. 2017]: Questão motriz; Investigações; Ferramentas tecnológicas; Colaboração.

Aproximando para os cursos de engenharia, a aprendizagem baseada em projetos (ABP) objetiva fazer a interlocução da vida real com os conceitos teóricos e tecnológicos. Para fazer essa relação, é importante incorporar uma visão da educação em que os alunos sejam participantes ativos na construção do seu conhecimento, o que fomenta os entendimentos sobre o construtivismo e o interacionismo. Ao explanar os cinco princípios da $\mathrm{ABP}$, que são a proposição orientada de um problema; a organização do projeto; a 
VIII Congresso Brasileiro de Informática na Educação (CBIE 2019)

Anais do XXX Simpósio Brasileiro de Informática na Educação (SBIE 2019)

integração entre a teoria e a prática; a autonomia dos estudantes na tomada das decisões do projeto; e o estímulo ao trabalho em grupo, percebemos que a Aprendizagem Baseada em Projetos consegue integrar vários elementos, devido as possibilidades que a ABP proporciona ao emergir processos de questionamento, como também a interdisciplinaridade e a flexibilidade cognitiva em ambientes educacionais [de Graaff and Deboer 2015].

Há uma série de benefícios que podem ser encontrados na literatura sobre os efeitos da ABP. Mesmo que haja muitas variações entre as propostas de ABP, um resultado com a qual ela geralmente está associada é o aumento da motivação dos estudantes para a aprendizagem [Thomas 2000], [Kolmos and de Graaff 2017], [Blumenfeld et al. 1991], [Boaler 1999]. Existem alguns desafios na adoção da ABP, alguns dos quais tem o poder de frear sua expansão [Blumenfeld et al. 1991]. A natureza construtivista e centrada no aluno da ABP é muito diferente da forma como são conduzidas as instruções que costumamos chamar de "tradicionais" e essa mudança de paradigma afeta as crenças dos professores sobre como a pedagogia deve ser [Condliffe et al. 2017], [Grant and Branch 2005].

\section{Aplicação da metodologia ABP}

A Faculdade UnB Gama da Universidade de Brasília (UnB) provê uma entrada única para os seus cursos de Engenharia. O projeto pedagógico dos cursos determina que todos os estudantes cursem disciplinas iguais e comuns aos cursos até o terceiro semestre, chamadas de disciplinas do "Tronco Comum". A partir do terceiro semestre, os estudantes devem escolher dentre as cinco engenharias existentes, a saber: Aeroespacial, Automotiva, Eletrônica, Energia e Software, para a partir da opção escolhida, cursar as próximas disciplinas constantes do projeto pedagógico do curso escolhido. Todos esses cursos de Engenharia foram reconhecidos pelo Ministério da Educação (MEC) em avaliações formais realizadas pelo Instituto Nacional de Estudos e Pesquisas Educacionais Anísio Teixeira (INEP).

A disciplina usada trata da integração entre engenharias existentes na faculdade UnB Gama da universidade de Brasília (UnB). Segundo sua ementa, tem como objetivo principal "preparar o estudante para futuras disciplinas profissionalizantes, incluindo competências às atividades interdisciplinares relativas à profissão de engenheiro". A faculdade oferece 100 novas vagas por semestre para cada curso e esta disciplina é obrigatória e ofertada no quarto semestre letivo, logo após a opção dos estudantes pelas sua Engenharias específica. São ofertadas 05 turmas por semestre, com uma média de 20 a 30 alunos por turma, ou seja, no total a um contingente de mais de 100 alunos por semestre, formado por alunos das 5 engenharias da faculdade, assim como o conjunto de professores alocados na disciplina também é formado por um docente de cada engenharia. Os alunos podem se matricular em qualquer turma da disciplina, pois terão acesso a todos os professores de todas as turmas. O professor de cada turma é tido como o orientador dos projetos da sua respectiva turma, embora seja possível consultar os outros professores das outras turmas, quanto às suas respectivas especialidades de Engenharias. Segundo o cronograma das aulas da disciplina, existem encontros com a participação dos alunos de todas as turmas e encontros onde cada turma se reserva aos trabalhos próprios de seus projetos particulares. O planejamento da aplicação da metodologia ABP ocorreu em 4 grandes fases, conforme apresentado na Figura 1. As fases são: 
VIII Congresso Brasileiro de Informática na Educação (CBIE 2019)

Anais do XXX Simpósio Brasileiro de Informática na Educação (SBIE 2019)

Fase 1 - Iniciação : Em que são estabelecidas as definições preliminares e é delimitado o escopo inicial dos projetos a serem desenvolvidos. Inicialmente é apresentado aos estudantes o plano de ensino, estruturado segundo a metodologia ABP. Em seguida, os professores divulgam os temas dos projetos e solicitam que as equipes discutam a apresentem propostas de projetos integradores para resolver problemas reais envolvendo conhecimentos de todas as engenharias. Nesta fase são formadas as equipes, com estudantes de cada turma, usando as Engenharias da faculdade (Aeroespacial, Automotiva, Eletrônica, Energia e Software). Concomitantemente são dadas aulas, ministradas pelos professores sobre os conteúdos teóricos necessários à elaboração de projetos de engenharias. Os marcos desta fase são: a) Uma prova do conteúdo teórico ministrado, realizada usando recursos do (Moodle), a ferramenta adotada pela instituição para todas as suas disciplinas. A prova possui questões de múltipla escolha sorteadas randomicamente de um banco de questões previamente definido. A correção é feita pela ferramenta, facilitando a aplicação da prova em uma disciplina com mais de 100 alunos em média, em suas turmas, realizada em um auditório com capacidade compatível com grandes turmas de disciplinas; b) O Ponto de Controle 1 (PC1 - Figura 1),em que é esperada a entrega de um relatório com a proposta de projeto integrador contendo o planejamento inicial do projeto com problema, objetivos geral e específicos, escopo preliminar e estrutura analítica do projeto, além da composição da equipe, as responsabilidades das frentes de trabalho com alunos de uma mesma engenharia, suas formas de comunicação, análise de riscos do projeto e estratégia para o acompanhamento do projeto. Neste ponto de controle as propostas de projeto são apresentadas para uma banca composta pelo professor orientador e pelos demais professores, representando todas as engenharias envolvidas. Durante as apresentações os professores fornecem feedbacks sobre todos os projetos com comentários sobre os pontos fortes, fracos e sugestões de melhoria, além de questionamentos que façam os alunos refletirem sobre os riscos técnicos dos seus projetos.

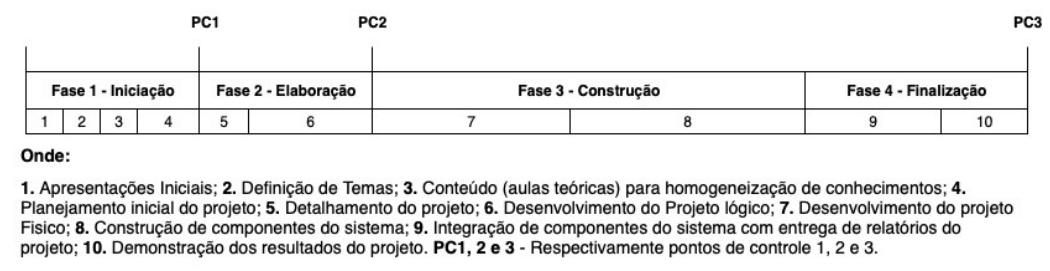

Figure 1. Fases da disciplina

Fase 2- Elaboração : As análises das críticas e recomendações oriundas do PC1 geram ajustes nos projetos que devem ser incorporados nos relatórios dos próximos pontos de controle. Os estudantes passam então para a fase de elaboração, cujo foco está no detalhamento do projeto e na elaboração do projeto lógico. O detalhamento do projeto trabalha sobre pontos como o detalhamento dos requisitos funcionais e não funcionais e a escolha de quais artefatos serão usados para demonstrar os resultados do projeto (fase de finalização). O projeto lógico tem também como foco identificar as teorias que serão necessárias às suas execuções. Nesse sentido devem ser identificados e estudados os métodos, técnicas, ferramentas e formas de montagem necessárias para a próxima fase (construção). Nesta fase podem ser também necessários alguns experimentos ou ensaios práticos em laboratórios sobre pontos específicos do projeto, para escolher as melhores opções possíveis de acordo com os prazos da disciplina, os recursos materiais, e con- 
VIII Congresso Brasileiro de Informática na Educação (CBIE 2019)

Anais do XXX Simpósio Brasileiro de Informática na Educação (SBIE 2019)

hecimento que os alunos devem adquirir sobre assuntos específicos para o projeto. Os experimentos podem ser sugeridos pelos professores e o acesso a laboratórios se dá sobre a orientação deles ou técnicos alocados em laboratórios específicos, conforme disponibilidade da instituição. A culminância desta fase ocorre com a entrega do relatório do segundo ponto de controle contendo a apresentação do projeto lógico.

Fase 3 - Construção: Nesta fase o projeto passa por novos detalhamentos onde cálculos são demonstrados conforme os métodos e técnicas previstas na etapa 2 , assim como o uso das ferramentas escolhidas. Nesta fase, todas as estruturas e partes do projeto são montadas e integradas para satisfazerem a solução pretendida. Além disso, são feitos testes para avaliar o funcionamento do artefato que será usado na próxima fase para demonstrar o projeto funcionando. O produto final é preparado para a entrega final e respectivas demonstrações exigidas na próxima fase (finalização). Neste ponto o relatório final do projeto já se encontra em andamento, para ser totalmente finalizado para entrega no ponto de controle 3 (PC3), ao final da fase 4.

Fase 4 - Finalização : Nesta fase, o relatório final do projeto é concluído e entregue, assim como os produtos de demonstração são integrados, consolidados a partir das correções dos problemas descobertos nos testes realizados anteriormente. Todo esse material fica pronto para ser observado pelos professores orientadores e participantes das outras turmas da disciplina, em apresentações previamente planejadas. Na Seção 4 são exemplificados alguns projetos e relatadas as principais dificuldades enfrentadas por professores e estudantes ao executarem.projetos envolvendo as 5 engenharias.

\section{Relato de Experiência e Análise dos Resultados}

Esta pesquisa apresenta um relato de experiência e análise dos resultados obtidos com os projetos realizados na disciplina desde o primeiro semestre de 2018 até o primeiro semestre de 2019. No total foram realizados 20 projetos,cujas análises foram feitas a partir dos acompanhamentos das turmas ao longo de cada semestre letivo. Elas contêm as dificuldades vivenciadas nos projetos sob o ponto de vista dos professores, ou seja, são resultantes dos acompanhamentos diários das turmas e dos pontos de controle com os alunos e todos os professores. Cada projeto gerou uma entrega final de um protótipo funcional, afim de demonstrar seus resultados. Nos pontos de controle os resultados parciais ou finais foram apresentados para uma banca formada pelos professores das 5 turmas e, portanto, das 5 engenharias existentes na faculdade. Os relatórios dos projetos são entregues pela ferramenta Moodle e as apresentações são realizadas em eventos previamente agendados, segundo o cronograma de aulas da disciplina, entregue no primeiro dia de aula. Todos os integrantes das turmas devem participar destes eventos e os feedbacks dos professores são dados após as apresentações.

Nos feedbacks são identificados os problemas, pontos fortes e fragilidades dos projetos, além de sanadas as dúvidas dos estudantes e professores sobre pontos específicos de cada projeto, tais como forma de condução do projeto, itens que necessitam de maiores esclarecimentos ou estudos mais aprofundados, dentre outros. Os resultados dessas análises e discussões consubstanciam atribuições de notas para as equipes de cada turma para cada ponto de controle (PC1, PC2 e PC3). As notas dos pontos de controle, as notas da prova individual realizada após o ciclo de aulas teóricas iniciais, e a avaliação dos próprios estudantes sobre os integrantes das suas equipes de projeto constituem os termos da fórmula 
VIII Congresso Brasileiro de Informática na Educação (CBIE 2019)

Anais do XXX Simpósio Brasileiro de Informática na Educação (SBIE 2019)

de cálculo usada na avaliação de desempenho dos estudantes. Ao final, esse cálculo gera a nota da turma e, consequentemente, a menção final atribuída conforme os regulamentos da instituição. Analisando os problemas percebidos em relação a percepção dos professores foi possível agrupa-los em classes, como as que se seguem:

1) Problemas relacionados ao estabelecimento, gestão e acompanhamento dos projetos de Engenharias como uso de modelos de ciclo de vida, delimitação de escopo e formas de gestão de projetos de engenharias. Dentre eles podem ser ressaltados questões como: a) Dificuldades em estabelecer e delimitar um problema dentro do tema do projeto. Atualmente os estudantes têm sido instigados a pesquisar os temas em busca de questões não totalmente respondidas ou cujas respostas, na opinião deles, ainda possam ser melhoradas ou modernizadas. Uma sugestão usada para fomentar melhorias neste item é solicitar aos estudantes propor uma questão de pesquisa que, segundo eles pode ser solucionada por um projeto envolvendo todas as engenharias. Além disso, uma pergunta importante a ser respondida por eles para os problemas propostos é "qual a responsabilidade de cada engenharia no projeto e no produto final almejado?”; b) Dificuldades em esclarecer os objetivos do projeto de engenharia. A partir do problema a discussão é direcionada ao objetivo geral do projeto e a quais passos deveriam ser realizados para satisfazer o objetivo geral. Essa discussão é importante para melhorar a organização de estudantes, ainda iniciantes nas suas respectivas engenharias, sobre o que deve ser feito (passos do projeto), as responsabilidades e as necessidades de aquisição de conhecimentos. Nesse raciocínio objetivos geral e específicos têm sido derivados; c) Alto nível de abstração dos requisitos do projeto. Tem sido observada uma dificuldade dos estudantes em detalhar os requisitos dos produtos resultantes do projeto em um nível de abstração suficiente para que sejam implementados. Um exemplo de requisito inicial: " $O$ sistema devem conter dispositivos sensores para identificar....”, sem uma descrição mais precisa do que deve ser identificado, medido ou controlado, além de quais sensores são necessários. Ademais, percebeu-se uma certa confusão entre requisitos funcionais e não funcionais. Por exemplo, o requisito: "o motor deve ser capaz de executar seu trabalho com excelência...”, sem uma definição mais precisa sobre qual o trabalho a ser executado, o tempo de execução e a definição do que seria considerado "excelência". Considerando que o conjunto de requisitos tanto funcionais, quanto não funcionais, constituem ao final o escopo do projeto a ser estabelecido, os estudantes têm sido questionados a respeito do que eles entendem por termos usados nos seus relatórios (excelência, por exemplo). Para melhorar este aspecto, foi incluído o tema sobre requisitos nas aulas teóricas ministradas no início da disciplina, alertando para que os requisitos sejam detalhados no ponto onde sejam úteis para as implantações pretendidas. Desta forma, mesmo sendo requisitos iniciais, eles devem apoiar o raciocínios sobre quais atividades devem ser realizadas para satisfaze-los. Além do mais, as atividades identificadas devem fazer parte da Estrutura Analítica do Projeto (EAP), ela apoia a elaboração do planejamento do projeto e estabelecimento do seu cronograma. Neste tipo de procedimento, os problemas identificados pelos estudantes ao longo das atividades necessárias são tratados como riscos do projeto a serem mitigados ou contingenciados no caso de suas ocorrências. Por exemplo, um risco comum envolve conhecimentos técnicos não disponíveis na equipe. A busca por eles se dá usualmente com a ajuda do professor orientador da turma. Dessa forma, é mostrado aos estudantes a importância de alinhar os requisitos às atividades no projeto, seu cronograma, a alocação de recursos e a execução de estimativas de prazos para realiza- 
VIII Congresso Brasileiro de Informática na Educação (CBIE 2019)

Anais do XXX Simpósio Brasileiro de Informática na Educação (SBIE 2019)

las. Teoricamente o assunto é abordado nas aulas iniciais e, na prática dos projetos. d) Ociosidade de integrantes da equipe. Percebeu-se uma certa ociosidade de alguns estudantes da disciplina devido ao tamanho das equipes constituídas a partir de turmas entre 20 e 30 alunos. Ao invés de limitar vagas nas turmas, o que não seria possível haja vista o contingente de alunos candidatos à disciplina, a proposta deste primeiro semestre de 2019 foi subdividir as turmas em dois projetos por turma e, consequentemente equipes variando entre 10 e 15 alunos. O semestre está em andamento, mas já foram verificadas melhorias nas alocações das equipes, além de melhorias tanto no acompanhamento dos projetos, quanto nas participações e contribuições dos seus elementos. Neste caso o professor orientador da turma é importante para manter a homogeneização de elementos de engenharias diferentes.

2) Problemas relacionados aos métodos e técnicas necessários à execução do projeto de engenharia: Falta de conhecimento sobre métodos técnicas ou uso de ferramentas, pois os alunos da disciplina estão ainda no início dos seus cursos em que pode ocorrer a falta de conhecimento sobre algum tipo de método, técnica ou mesmo uso de ferramentas. Neste ponto é grande a importância da ação do professor orientador para direcionar os alunos nesses estudos. São exemplos de problemas técnicos: projeto e cálculos de estruturas, projetos e dimensionamento de motores elétricos, baterias e placas fotovoltaicas, estudos de mecânica dos fluidos, termodinâmica, projeto de arquiteturas de software, linguagens, plataformas operacionais, uso de software embarcado, uso de equipamentos eletrônicos como arduíno, raspberry, projeto de placas eletrônicas, projeto de engrenagens, dentre inúmeros outros já ocorridos e certamente ainda a ocorrer devido à multiplicidade de projetos. Pode acontecer também a necessidade de conhecimentos fora da área de atuação do professor orientador. Mesmo assim, neste caso é importante a participação deste professor para facilitar o acesso dos estudantes a pessoas que detenham este conhecimento ou a laboratórios restritos. Isso porque teoricamente todos os discentes e técnicos da instituição estão disponíveis para consultas sobre suas especialidades. Exemplos destes tipos de busca são: atividades que exigem soldas de partes de estruturas, ou serviços de torno de peças em laboratórios avançados da faculdade.

3) Problemas relacionados à documentação dos projetos (relatórios e apresentações): Documentar um projeto de engenharia não é uma tarefa trivial, principalmente para o estágio no curso onde os estudantes desta disciplina se encontram. As dúvidas residem principalmente em como estruturar o relatório do projeto de forma que ele fique inteligível aos leitores e apresente todo o seu conteúdo de forma coerente com o seu objetivo. Neste sentido, dificuldades mais comuns são encontradas em construir uma linha lógica para a documentação do projeto. Defeitos mais frequentes são: falta de uma verificação final antes de entregar o documento, assuntos pulverizados em várias seções dos relatórios, figuras pouco legíveis na tentativa de fazer com que diagramas ou gráficos caibam no documento (ex.: EAPs diminuídas em tamanho até prejudicarem as suas leituras e entendimentos). Como a disciplina tenciona também desenvolver a capacidade escrita dos estudantes, uma forma de amenizar esses defeitos foi reforçar a orientação aos estudantes no decorrer do andamento da disciplina. Por exemplo, solicitando cópias prévias dos relatórios que serão entregues para avaliação preliminar dos professores. Outra dificuldade reside em construir as apresentações obrigatórias para cada ponto de controle. Os estudantes demonstram dificuldades em organizar os assuntos das apresentações, distribuir os temas pelos seus integrantes e cumprir com o tempo 
VIII Congresso Brasileiro de Informática na Educação (CBIE 2019)

Anais do XXX Simpósio Brasileiro de Informática na Educação (SBIE 2019)

disponível. Neste sentido, além das orientações específicas de cada professor orientador para a sua própria turma, são de alta importâncias as sessões de feedbacks realizadas após os pontos de controle onde os estudantes tomam contato com as opiniões de outros professores, além do seu orientador.

Em relação a percepção dos estudantes envolvidos na aplicação da $\mathrm{ABP}$ foram identificados alguns desafios da disciplina PI1, os quais são: 1) Gestão do projeto integrador: os projetos são gerenciados pelos próprios estudantes que ainda não têm experiência em gestão de projetos. Logo, isso pode gerar problemas de gestão e conflitos entre os integrantes das equipes. Neste aspecto, destaca-se ainda que delegar tarefas que sejam condizentes com a engenharia específica de cada integrante da equipe, falta de envolvimento e comprometimento de alguns estudantes também são relatados como dificultadores. 2) Multidisciplinaridade: os estudantes encontram dificuldades em definir um projeto que agregue conhecimento de todas as engenharias, quando cada membro da equipe só tem familiaridade com a sua própria engenharia. Isso pode complicar a identificação de pontos de intersecção entre as áreas de conhecimento. 3) Gestão da disciplina: alguns estudantes relatam dificuldades em identificar com clareza o que é necessário entregar em cada ponto de controle da disciplina. Ademais, PI1 é uma disciplina que demanda muito tempo e dedicação dos estudantes, o que torna difícil a conciliação das demandas desta disciplina com as outras demandas da faculdade.

\section{Considerações Finais}

Neste trabalho foi apresentado um relato da experiência da adoção da ABP em uma disciplina que integra estudantes de várias engenharias visando solucionar um problema da vida real, por meio de um projeto integrador de engenharias. A experiência adquirida com os resultados motiva a continuidade do uso da ABP na disciplina devido às opiniões de docentes e discentes, com a adoção das sugestões de melhorias coletadas até o momento. Como trabalhos futuros, espera-se que sejam expandidas as pesquisas de opinião sobre a percepção dos alunos sobre a disciplina, seus desafios e formas de condução. Além disso, pensa-se no uso de alguma ferramenta para a elaboração de uma Wiki em que o conteúdo dos projetos sejam disponibilizados para a comunidade acadêmica, Além dos relatórios dos projetos apresentados nos pontos de controle seriam também disponibilizados códigos, cálculos e documentos elaborados pelos próprios alunos. Este repositório comportaria exemplos e experiências de outros semestres, podendo servir inclusive como fontes de ideias para próximos projetos da disciplina.

\section{References}

Adderley, K. et al. (1975). Project methods in higher education, volume 24. Society for research into higher education.

Bender, W. N. (2012). Project-based learning: Differentiating instruction for the 21st century. Corwin Press, Thousand Oaks, California, USA.

Blumenfeld, P. C., Soloway, E., Marx, R. W., Krajcik, J. S., Guzdial, M., and Palincsar, A. (1991). Motivating project-based learning: Sustaining the doing, supporting the learning. Educational psychologist, 26(3-4):369-398.

Boaler, J. (1999). Mathematics for the moment, or the millennium. Education Week, 17(29):30-34. 
VIII Congresso Brasileiro de Informática na Educação (CBIE 2019)

Anais do XXX Simpósio Brasileiro de Informática na Educação (SBIE 2019)

Condliffe, B., Quint, J., Visher, M. G., Bangser, M. R., Drohojowska, S., Saco, L., and Nelson, E. (2017). Project-Based Learning A Literature Review Working Paper Prepublication copy: Release. Working paper.

de Graaff, E. and Deboer, J. (2015). Capacity building for engineering education. In 2015 International Conference on Interactive Collaborative Learning (ICL), pages 10221025. IEEE.

Diehl, W., Grobe, T., Lopez, H., and Cabral, C. (1999). Project-based learning: A strategy for teaching and learning. Boston: Center for youth development and education.

Grant, M. M. and Branch, R. M. (2005). Project-based learning in a middle school: Tracing abilities through the artifacts of learning. Journal of Research on Technology in Education, 38(1):65-98.

Helle, L., Tynjälä, P., and Olkinuora, E. (2006). Project-Based Learning in PostSecondary Education - Theory, Practice and Rubber Sling Shots. Higher Education, 51(2):287-314.

Jones, B. F., Rasmussen, C. M., and Moffitt, M. C. (1997). Real-life problem solving: A collaborative approach to interdisciplinary learning. American Psychological Association.

Knoll, M. (1997). The Project Method: Its Origin and International Development. Progressive Education Across the Continents., 27(September 1996):40-44.

Kolmos, A. and de Graaff, E. (2017). Problem-based and project-based learning in engineering education. In Johri, A. and Olds, B. M., editors, Cambridge Handbook of Engineering Education Research, pages 141-160. Cambridge University Press.

Krajcik, J. S., Blumenfeld, P. C., Marx, R. W., and Soloway, E. (1994). A collaborative model for helping middle grade science teachers learn project-based instruction. The elementary school journal, 94(5):483-497.

Mahasneh, A. M. and Alwan, A. F. (2018). The effect of project-based learning on student teacher self-efficacy and achievement. International Journal of Instruction, 11(3):511524.

Martins, V. F., Sampaio, P. N., Cordeiro, A. J. A., and Viana, B. F. (2017). Problem-based learning methodology applied within a data network infrastructure design course a real case implementation. In 2017 12th Iberian Conference on Information Systems and Technologies (CISTI), pages 1-6. IEEE.

Morgan, A. (1975). Theoretical Aspects of Project- Based Leurning in Higher Education. British Journal of Educational Technology, 14(1):66-78.

Morgan, A. (1983). Theoretical aspects of project-based learning in higher education. British Journal of Educational Technology, 14(1):66-78.

Moursund, D. G. (1999). Project-based learning using information technology. International society for technology in education Eugene, OR.

Thomas, J., Michaelson, A., and Mergendoller, J. (1999). Introduction to project based learning. Buck Institute for Education Project Based Learning Handbook.

Thomas, J. W. (2000). A Review of Research on Project-Based Leaming. Working paper. 